\title{
Le long cheminement de la mémoire collective irlandaise : A Long Long Way de Sebastian Barry (2005)
}

\section{Sylvie Mikowski}

\section{(2) OpenEdition \\ Journals}

Édition électronique

URL : http://journals.openedition.org/etudesirlandaises/2172

DOI : $10.4000 /$ etudesirlandaises. 2172

ISSN : 2259-8863

Éditeur

Presses universitaires de Rennes

Édition imprimée

Date de publication : 30 juin 2011

Pagination : 121-130

ISBN : 978-2-7535-1348-8

ISSN : 0183-973X

Référence électronique

Sylvie Mikowski, « Le long cheminement de la mémoire collective irlandaise : A Long Long Way de Sebastian Barry (2005) », Études irlandaises [En ligne], 36-1 | 2011, mis en ligne le 30 juin 2013, consulté le 03 mai 2019. URL : http://journals.openedition.org/etudesirlandaises/2172 ; DOI : 10.4000/etudesirlandaises.2172

Ce document a été généré automatiquement le 3 mai 2019.

(c) Presses universitaires de Rennes 


\title{
Le long cheminement de la mémoire collective irlandaise : A Long Long Way de Sebastian Barry (2005)
}

\author{
Sylvie Mikowski
}

1 L'Irlande, pour des raisons qui ont été démontrées maintes fois, offre un exemple frappant d'une part d'une concurrence entre mémoire et histoire, d'autre part d'une mémoire sélective et forcée, mise au service d'une idéologie visant à construire ou pour le moins renforcer une identité nationale, pour reprendre les termes utilisés par le philosophe Paul Ricœur dans La mémoire, l'histoire, l'oubli'. Dans cette perspective, les commémorations, jouent un grand rôle, celles qui ont lieu autant que celles qui n'ont pas lieu. Ce n'est ainsi pas un hasard si ce furent les cérémonies de commémoration du $50^{\mathrm{e}}$ anniversaire du Soulèvement de Pâques de 1916 qui marquèrent l'émergence d'un mouvement protestataire parmi les intellectuels, les historiens et les écrivains, identifié depuis comme "le courant révisionniste", qui provoqua lui-même l'apparition d'un courant "contre-révisionniste». Aux yeux de certains commentateurs, les grandioses cérémonies de 1966, avec parade militaire et défilé de chars blindés, orchestrées par le parti Fianna Fail, auraient joué un rôle non négligeable dans le déclenchement des troubles en Irlande du Nord, par leur exaltation de la violence armée et du sacrifice de quelques-uns pour une cause dite commune.

2 Pâques 1916 appartient certainement à la catégorie des événements historiques irlandais ayant reçu le plus grand nombre d'interprétations variées et totalement contradictoires. Tandis que le gouvernement irlandais a voulu en faire le symbole de la naissance de la nation, un des moments forts de la commémoration étant la lecture du texte de la proclamation de la république irlandaise lue par Padraig Pearse le 24 avril 1916, des historiens, au premier rang desquels Roy F. Foster, y voient pour leur part une série d'erreurs et de bévues, la principale étant le fait même que la rébellion ait eu lieu². La Raven Arts Press de Dermot Bolger publia pour sa part en 1986 un pamphlet intitulé : 16 on 16: Irish Writers on the Easter Rising, dans lequel le journaliste du Irish Times Fintan O'Toole décrit les commémorations du soulèvement comme une tentative de transformer 
un échec en une action glorieuse et mémorable ${ }^{3}$. La controverse a également inspiré à Roddy Doyle, appartenant à la même mouvance esthétique et idéologique que Dermot Bolger, un roman intitulé A Star Called Henry ${ }^{4}$, dans lequel il remet en question l'orthodoxie nationaliste concernant les buts et les enjeux du Soulèvement. En tout cas, bien que contestée, la commémoration de Pâques 1916 n'en constitue pas moins un exemple de ce que Paul Ricœur nomme une mémoire forcée, "armée », comme il la décrit, "par une histoire elle-même "autorisée", l'histoire officielle, l'histoire apprise et célébrée publiquement ${ }^{5}$ ».

3 Mais surtout, la mémoire imposée de Pâques 1916 va de pair avec ce que Ricœur appelle un oubli forcé : celui des milliers d'Irlandais qui au moment du Soulèvement se battaient contre les Allemands dans les tranchées en Belgique et en France, sous l'uniforme de l'armée britannique. La participation de 200000 Irlandais à la Première Guerre mondiale, qui causa la mort de 35000 d'entre eux, ne donna lieu qu'à des commémorations furtives jusqu'en juillet 2006, date de la première commémoration officielle en République d'Irlande de la Bataille de la Somme. En revanche, 200 vétérans de cette guerre furent assassinés entre 1919 et 1922, accusés de collusion avec "l'ennemi », c'est-à-dire les Anglais. En 1927, une proposition faite aux députés du Dáil d'ériger un monument aux morts à Merrion Square fut vigoureusement repoussée. Les cérémonies du 11-Novembre, ou Remembrance Day, furent longtemps associées en République d'Irlande à des manifestations de soutien aux unionistes, d'autant plus qu'on y jouait le God Save the Queen et qu'on y arborait le drapeau de l'Union Jack. Le jardin de Islandbridge, seul mémorial de la Première Guerre mondiale autorisé à Dublin, tomba pendant des années en déshérence.

4 Le roman de Sebastian Barry, A Long Long Way ${ }^{6}$, illustre de manière remarquable d'une part le lien entre récit de fiction et récit historique, d'autre part entre mémoire et histoire, et pose la question du rôle de la littérature dans la transmission d'une mémoire collective. Il se trouve que le roman enchevêtre trois formes de mémoire, ou plutôt cherche à réparer trois formes d'oubli : celui, collectif, du rôle des Irlandais engagés dans l'armée britannique pour se battre dans les Ardennes, la Flandre et la Somme; celui ensuite qui concerne l'histoire personnelle de la famille de Sebastian Barry. En effet, un de ses grands-pères combattit dans l'armée britannique pendant la Seconde Guerre mondiale, et surtout un de ses arrière-grands-pères était le Chief Superintendent de la Dublin Metropolitan Police, chargé de réprimer la Grande Grève de 1913. Il fut l'homme qui mena la charge au cours de laquelle quatre manifestants furent tués. Barry ressuscite cet embarrassant aïeul dans son œuvre sous les traits de Thomas Dunne, qui apparaît d'abord vieux et malade dans The Steward of Christendom ${ }^{7}$ puis est remémoré longtemps après sa mort par sa fille Annie dans Annie Dunne ${ }^{8}$, pour enfin être représenté dans la force de l'âge, veuf élevant seul ses quatre enfants, dans A Long Long Way. C'est là la troisième forme de réparation de l'oubli opérée par Barry au fur et à mesure que son œuvre se construit : il sort de l'ombre des personnages qui jusque-là n'avaient fait que hanter ses récits antérieurs, Willie apparaissant effectivement comme un fantôme sur la scène dans The Steward. De même, Barry recrée aux côtés de Willie le personnage de Jessie Kirwan, fils de Fanny et de Patrick Kirwan, protagonistes de sa pièce Prayers of Sherkin 9 .

5 En choisissant ainsi un membre différent de la même famille comme centre de conscience pour chaque œuvre Thomas, Annie ou Willie Dunne, Fanny ou Jessie Kirwan Barry suggère la variabilité des souvenirs et interroge le lien entre mémoire individuelle et mémoire collective : sont-elles discordantes ou nécessaires l'une à l'autre? N'existe-t-il de 
souvenirs que partagés? La vérité de Thomas se trouve-t-elle dans le souvenir que garde de lui sa fille Annie ou dans les souvenirs que sa mémoire à lui conserve des événements passés ? Et quelle part de mémoire, personnelle ou collective, peut ou doit contribuer à l'élaboration de l'histoire nationale? On retrouve là une autre problématique propre à Paul Ricœur, celle d'être pour soi-même ou/et pour les autres. Barry l'illustre à sa façon à travers la saga des Dunne, citoyens condamnables aux yeux des autres membres de la communauté nationale, car placés à contre-courant de l'idéologie du moment, soient parce qu'ils étaient catholiques du " Château ", c'est-à-dire loyalistes, juste au moment où l'union était sur le point de se dissoudre, ou parce qu'ils étaient soldats engagés dans l'armée britannique, appelés à tirer sur les rebelles de 1916, ou encore femmes célibataires sans enfant dans un pays qui sacralise le mariage et la maternité, comme Annie. En imaginant et en représentant verbalement la conscience, les souvenirs et le point de vue de ces personnages, en révélant leur "soi-même ", Barry confronte leur vérité intérieure à celle qui est construite par les autres.

6 Par ailleurs, l'œuvre de Barry met à contribution la mémoire du lecteur ou du spectateur, qui, en retrouvant des personnages rencontrés précédemment, mais aussi des faits passés avérés, est amené à se remémorer simultanément des récits fictifs et des récits historiques, expérience qui souligne l'identité de nature, purement verbale, entre histoire et fiction. A Long Long Way est ainsi accompagné d'une bibliographie des ouvrages historiques les plus récents publiés sur le rôle de l'Irlande dans la Grande Guerre, et s'appuie largement sur des faits authentiques. Willie Dunne est le représentant fictif des 80000 Irlandais qui s'engagèrent au cours des douze premiers mois de la guerre, formant trois brigades, dont l'une était composée uniquement de soldats venant d'Ulster, la célèbre $36^{\mathrm{e}}$ Division. Willie appartient pour sa part au régiment des Royal Dublin Fusiliers, une composante de la $16^{\mathrm{e}}$ division. Il participe à la bataille de Saint-Julien, près d'Ypres, au cours de laquelle le $2^{\mathrm{e}}$ bataillon des Fusiliers de Dublin fut presque entièrement exterminé, empoisonné par le fameux gaz moutarde déversé par les Allemands que personne ne connaissait encore, épisode reconstitué dans le chapitre 4 du roman. Il est tout à fait exact que, comme Willie de retour à Dublin en permission, un nombre important de soldats du régiment des Fusiliers de Dublin prirent part à la répression du Soulèvement du 24 avril 1916 alors qu'ils étaient en permission. De retour au front, Willie participe avec son régiment à la prise de Guillemont et de Guinchy, au cours de laquelle les bataillons de l'Ulster et ceux du Sud combattirent côte à côte, et où 4314 Irlandais perdirent la vie. Puis Willie prend part à la bataille de Messines, qui dura plus d'une semaine, victoire à laquelle la $16^{\mathrm{e}}$ division d'Ulster et la $36^{\mathrm{e}} \mathrm{du}$ Sud contribuèrent ensemble, et au cours de laquelle Willie Redmond, le frère de John, le leader du Irish Parliamentary Party, fut tué, comme le rapporte Barry dans le chapitre 18. Willie lui-même est tué lors des derniers combats de 1918, le $2^{\mathrm{e}}$ régiment des Fusiliers de Dublin ayant effectivement été engagé en octobre 1918 dans la bataille du Cateau dans laquelle $44 \%$ de ses effectifs furent tués.

7 Le personnage de Father Buckley, qui dans le roman de Barry prie avec les soldats avant la bataille et donne l'extrême-onction aux mourants, est sans doute inspiré du Révérend Willie Doyle, figure légendaire connue pour son courage, qui donnait les derniers sacrements aux soldats blessés à mort dans les circonstances les plus dangereuses. Fervent admirateur de Redmond, il exhortait les soldats à combattre non seulement pour défendre la Belgique catholique mais aussi la cause de l'Irlande, à travers celle d'une autre nation, comme il le disait. Il fut tué à Ypres en 1916, comme l'est Father Buckley dans le 
roman. Le sort de Jesse Kirwan, exécuté pour rébellion, rappelle quant à lui celui des 239 soldats irlandais condamnés à mort et exécutés pendant la guerre par les autorités britanniques pour désertion ou désobéissance. Un rapport récent, publié seulement en 2004, a établi la preuve des préjugés racistes à l'encontre des Irlandais qui motivèrent ces jugements le plus souvent iniques et sans commune mesure avec la faute commise.

En ce qui concerne les motivations attribuées par Barry à ses personnages pour décider de s'engager dans l'armée, alors que le service militaire n'était pas obligatoire en GrandeBretagne en 1914, elles sont tout à fait conformes à ce que les historiens nous en disent aujourd'hui. Ils expliquent ainsi qu'il existait une longue tradition de l'engagement militaire chez les Irlandais; que certains d'entre eux s'étaient laissé convaincre au début de la guerre par John Redmond de la nécessité de se battre pour l'Irlande au sein de l'Empire, puisque le Home Rule était pratiquement acquis; que les Irlandais étaient sensibles au parallèle établi entre la situation de la Belgique, petite nation catholique, avec celle de l'Irlande. D'autres s'étaient engagés pour des raisons économiques, fuyant le chômage, d'autres encore recherchaient simplement l'aventure et l'expérience du combat et des armes. Quant à Willie Dunne, fils du Chief Superintendent de la Dublin Metropolitan Police, sa décision de s'engager s'impose à lui comme une évidence. Cependant, il est fait allusion au cours du récit à la chute spectaculaire du nombre de soldats irlandais enrôlés volontaires après le soulèvement de Pâques et suite à ce qu'on a nommé la «crise de la conscription ».

9 Ainsi, le roman de Barry est solidement adossé aux faits historiques attestés par les archives, statistiques, documents et témoignages de l'époque. Cependant, A Long Long Way n'est pas un livre d'histoire, même s'il peut s'apparenter par endroits à une reconstitution historique, mais avant tout un travail de mémoire, qui cherche à sortir de l'amnésie collective une page entière du passé, dont le souvenir a été effacé par un exemple particulièrement frappant d'oubli forcé. Ce sont donc les ressources de la mémoire que Barry utilise pour faire ressurgir des images enfouies et provoquer ainsi l'anamnèse. Dans ce but il a recours à l'imagination, dans la mesure où imagination et souvenir ont comme point commun de rendre présent ce qui est absent, une des fonctions de l'imagination, que Ricœur désigne comme sa "fonction ostensive ${ }^{10}$ " étant de "mettre sous les yeux ", de donner à voir, de faire voir. Il souligne également que le récit, qu'il soit historique ou fictif, donne à la fois à comprendre et à voir, qu'il existe un rapport de quasi identité entre lisibilité et visibilité.

Il n'est donc pas étonnant que parmi toutes les techniques propres à la fiction narrative, Barry ait privilégié dans A Long Long Way celle qui concerne la manipulation du point de vue. Le récit est en effet entièrement narré du point de vue de Willie, soldat ordinaire, que sa jeunesse, son ignorance, sa confiance dans son père, ainsi que son caractère simple et obéissant identifient au personnage type du naif ou de l'innocent qui va servir de conscience - témoin au lecteur pour découvrir à travers lui une situation incompréhensible, révoltante ou insupportable, procédé bien connu depuis Voltaire, Swift, Aldous Huxley ou encore Imre Kertezs dans Etre sans Destin. Ainsi, comme le souligne Christina Hunt Mahony dans un article intitulé « Barry's Naïfs and the Poetry of Humanism ${ }^{11}$ », Willie, comme tout soldat dans n'importe quelle armée, ne comprend pas toujours ce qu'on lui demande de faire. Le sentiment permanent de désorientation, d'incompréhension et d'ignorance qui l'accompagne tout au long des différentes batailles auxquelles il participe l'entraîne à ne se fier qu'à ses perceptions : «He had hardly a true 
idea who he was in that second, what he was thinking, where he was, what nation, he belonged to, what language he spoke » (35).

11 Le choix de raconter ainsi l'histoire à travers les perceptions de Willie se révèle particulièrement efficace dans deux épisodes: celui de la première attaque au gaz moutarde subie par le bataillon de Willie, et celui du Soulèvement de Pâques à Dublin. Dans ce dernier, l'incompréhension totale de Willie des scènes qui se présentent à ses yeux « it was the most astonishing thing Willie Dunne ever thought he would see in his native place» (87) souligne la confusion qui caractérisa l'événement. Ainsi Willie et ses camarades pensent un moment que des Allemands ont envahi Dublin, et que c'est contre eux qu'on les envoie se battre; Sackville Street, si familière à Willie, est soudain transformée à ses yeux en champ de bataille; un officier interdit à Willie de parler à "l'ennemi ", alors qu'il ne fait que poser une question à un citoyen dublinois d'apparence tout à fait ordinaire à ses yeux. Quelques instants plus tard, il reçoit dans ses bras un jeune rebelle agonisant qui, trompé par la couleur de son uniforme, le prend pour un Écossais. La plus grande confusion concerne peut-être le document distribué à Willie et qui fait référence à «our gallant allies in Europe » (90). L'expression est extraite du texte authentique de la Proclamation de la République irlandaise lue le 24 avril 1916 par Pearse et fait référence au soutien apporté à la rébellion par les Allemands: elle parait incompréhensible à Willie, qui se bat avec ses camarades contre ces mêmes Allemands en Flandre, au prix de souffrances extrêmes. Ainsi, la représentation d'une scène connue du point de vue d'un personnage qui en ignore tout produit un effet de défamiliarisation qui pousse le lecteur à abandonner ses souvenirs construits de l'événement pour le configurer autrement.

Dans l'épisode de l'attaque au gaz moutarde, les soldats, ignorant à nouveau tout de la nature de l'étrange nuage jaune qui se dirige vers leur tranchée, se basent uniquement sur leurs perceptions pour essayer de comprendre ce qui se passe. Odeurs et bruits se transforment bientôt en sensations horribles de douleurs et de suffocation : "Anyone that lingered tasted the smoke, felt the sharp tines in his throat raking and gashing, and he was undone » (47). Ce passage, qui mêle description des sensations éprouvées par les personnages, d'ordre surtout visuelles puisque la couleur jaune y joue un rôle essentiel, et évocations de l'apocalypse: "he thought horribly of the Revelation of St John and wondered if by chance and lack he had reached the unknown date of the end of the living world », (49), est à rapprocher d'un autre roman de guerre, The Red Badge of Courage de Stephen Crane. Ce récit de la Guerre de Sécession est très souvent loué pour son réalisme photographique, sa faculté de faire voir la réalité des batailles grâce au choix de la restriction du point de vue à travers la conscience du personnage principal, le soldat Henry Fleming. Le style de Barry, riche en images et en comparaisons qui frappent l'imagination, est également à rapprocher de celui de Crane, par exemple dans l'évocation de l'horreur éprouvée dans les deux récits par chacun des deux protagonistes à la vue des cadavres jonchant le champ de bataille. Seule l'ampleur de la dévastation et du massacre, infiniment plus grande entre 1914 et 1918 que pendant la Guerre civile américaine, semble séparer les deux guerres, comme l'atteste le parallèle suivant entre Crane et Barry :

He came to a fence and clambered over it. On the far side, the ground was littered with clothes and guns. A newspaper, folded up, lay in the dirt. A dead soldier was stretched with his face hidden in his arm. Farther off there was a group of four or five corpses keeping mournful company ${ }^{12}$.

[...] the sightless sockets peered at the living soldiers, the lipless teeth all seemed to have just cracked some mighty jokes. They were seriously grinning. Hundreds more 
were face down, and turned on their sides, as if not interested in such awful mirth, showing the gashes where missing arms and legs had been, their breasts turned away, and hundreds of floating hands, and legs, and big heavy puddles of guts and offal, all mixed through the loam and sharded vegetation. (Barry, 178) un cas, grinning dans l'autre, suggérant une inversion de l'animé et de l'inanimé qui provoque un effet grotesque. Les deux passages mentionnent également la position des cadavres allongés dans la saleté (the dirt) ou au milieu d'une végétation décomposée (loam and sharded vegetation) tels des détritus jonchant le sol, accentuant ainsi l'effet de déshumanisation recherché. Il est d'autant plus intéressant de rapprocher l'œuvre de Barry de celle de Crane dans la mesure où on a parlé du « révisionnisme » de ce dernier concernant l'histoire de la Guerre de sécession, et qu'il fut d'ailleurs taxé d'antipatriotisme quand le livre fut publié.

Mais surtout Crane comme Barry soulèvent la question de la nature du courage et de l'héroïsme, question également placée au cœur de l'historiographie de la période s'étendant entre 1916 et 1918 en Irlande. L'assomption sur laquelle repose la commémoration du Soulèvement de Pâques par l'État nationaliste irlandais est en effet celle de l'héroïsme des participants, censés avoir sacrifié leurs vies pour l'amour de la patrie. Même le célèbre poème de Yeats, «Easter 1916 ", malgré ses ambiguïtés, souligne comment l'événement éleva les hommes de 1916 à une hauteur dépassant le commun des mortels. Cette assomption en revanche fut niée par la force de l'oubli aux soldats irlandais morts pendant la Première Guerre. Or, de même que les historiens révisionnistes ont contesté l'héroïsme des rebelles de Pâques, préférant souligner leur manque de préparation et leur volonté de forcer le destin au nom d'un peuple qui ne leur demandait rien, de nombreux écrivains contemporains de la période qui suivit l'indépendance se sont également insurgés contre le culte de l'héroïsme entretenu par Yeats et le mouvement de la Renaissance celtique. L'esthétique de la Renaissance fut celle de l'épopée, du mythe, de la tragédie héroïque. passé glorieux ponctué d'exploits accomplis par des personnages exceptionnels ainsi que d'un âge d'or de la culture et de la vie nationale irlandaise, les auteurs de l'aprèsindépendance se tournèrent soit vers la satire, comme Flann O'Brien ou Sean O'Casey, soit vers le naturalisme, comme Patrick Kavanagh. À leur suite toute une génération de romanciers ou dramaturges, comme John McGahern ou Tom Murphy, s'est caractérisée par une peinture réaliste de l'anti-héroïsme et des vies étriquées et sans gloire de leurs personnages fictifs. L'œuvre de Barry semble s'inscrire directement dans ce mouvement de remise en cause d'une certaine forme d'écriture de l'histoire et de la littérature irlandaises. Ainsi dans A Long Long Way il privilégie l'homme ordinaire par rapport aux grands hommes, Willie se caractérisant d'ailleurs par sa petite taille, qui l'empêche de devenir policier comme son père mais pas de s'enrôler dans l'armée. Tout au long du récit, raconté donc du point de vue du soldat mais non par sa voix, le narrateur insiste sur le sentiment éprouvé par Willie de n'être qu'un élément infime et insignifiant d'un ensemble qui le dépasse : «It was then that he felt slight enough in the world, a creature of low stature » (49). Cette insistance sur la petitesse du protagoniste de Barry s'oppose au culte des héros qui parsème l'histoire d'Irlande, Daniel O'Connell étant surnommé "The Liberator ", Parnell «Ireland's Uncrowned King », Eamon de Valera "The Long Fellow » et Michael Collins «The Big Fellow». D'ailleurs la statue érigée à l'intérieur de la GPO à Dublin pour commémorer l'Insurrection de Pâques représente le géant Cúchulainn. 
Cependant la petite taille de Willie ne souligne pas seulement son aspect ordinaire et banal, mais aussi sa jeunesse, au point que son jeune âge ainsi que son ignorance et son innocence l'assimilent ni plus ni moins qu'à un enfant.

Ainsi nous apparaitt un autre aspect du récit, mise en lumière par les très nombreuses références religieuses qui imprègnent le récit. Il n'en est pour s'en convaincre que de citer la phrase : «Soon the places were filled with new men from home. Flocks and flocks and flocks of them, thought Willie. King George's lambs " (54). Ou encore : "The officer in charge, a florid-faced captain with a patch on one eye, lined them up, ready to embark. Willie remembered he used to be down here as a little fella with his father, to watch the Irish lambs being loaded on, for the English trade... » (85) Qu'ils soient agneaux destinés à l'immolation, ou enfants promis à un nouveau " Massacre des Innocents ", les soldats de Barry correspondent en tout point à la définition de la victime sacrificielle, désignée au hasard pour purger la communauté de ses pulsions de violence. C'est d'ailleurs son père qui envoie Willie au front, comme Abraham envoie son fils Isaac au sacrifice sur l'ordre de Dieu. Thomas en portera la culpabilité jusqu'à la fin de ses jours. Un des moments les plus émouvants du roman se produit lorsque Willie chante l'Ave Maria de Schubert devant ses camarades rassemblés, promesse d'une éternité après la mort qui selon les mots de Barry « could not be rendered meaningless even by slaughter» (134).

Ironiquement, Barry a donc recours dans ce roman à la même rhétorique du sacrifice que la littérature de la renaissance celtique. De même, le lyrisme de certains passages s'apparente au style de l'épopée, avec ses récits de batailles, d'exploits et de morts. Mais là où les nationalistes honorent le sacrifice des Insurgés de 1916, et lui donnent un sens en en faisant l'événement fondateur de la république, Barry laisse en suspens la question du sens du martyr des soldats irlandais de la Première Guerre, d'autant plus que son souvenir a été oblitéré. Par endroits, son roman semble répondre au poème de Yeats, et partager ses interrogations sur la nature et l'utilité du courage : "Was it needless death after all?»: en effet, un sacrifice non reconnu et qui n'est pas commémoré par la communauté est-il encore efficace, c'est-à-dire, comme le dit René Girard ${ }^{13}$, permet-il encore l'éradication de la violence et le maintien de la paix sociale? Au regard de la guerre civile qui déchira la société irlandaise après l'armistice de 1918 et le retour des soldats irlandais chez eux, ou encore des événements d'Irlande du Nord, conséquence directe du Soulèvement de Pâques, on peut en douter. Le vers dans lequel Yeats insiste sur la reconnaissance des intentions des rebelles de 1916 : «We know their dream; enough/ To know they dreamed and are dead » - prend une résonance ironique au regard des morts de Ypres, Messines et St-Julien, dont personne n'a souhaité pendant longtemps reconnaître pour quel idéal ils voulaient se battre et mourir. De même, le vers « Too long a sacrifice/Can make a stone of the heart " prend un sens tragiquement ironique si on l'applique au destin de Willie, qui traverse trois années de guerre avant de mourir, quelques semaines avant l'armistice, et dont Barry imagine l'épitaphe, qui constitue les derniers mots du roman, son existence se pétrifiant effectivement dans l'éternité de la mort et de cette inscription. Comme en réponse aussi au célèbre refrain de Yeats, "all changed, changed utterly ", Barry attribue la pensée suivante à Willie, de retour sur le front après sa permission à Dublin pendant laquelle il a tiré sur les insurgés de la GPO : " nothing had changed just where he found himself-utter change was just across the plains. Nothing had changed. But something had changed in Willie Dunne» (101), suggérant que le devenir du peuple irlandais se joua autant sur les champs de bataille de la Somme que dans Sackville Street. 
Peu à peu, la manière dont les Irlandais se souviennent de 1916 a également changé : les événements de Pâques et ceux de Ypres, Messines ou Gallipoli ont été "racontés autrement, en supprimant, en déplaçant les accents d'importance, en refigurant différemment les protagonistes de l'action en même temps que les contours de l'action ", pour reprendre la formule de Paul Ricœur dans La mémoire, l'histoire, l'oubli ${ }^{14}$. Le roman de Barry, de même que la pièce de Frank McGuiness, See the Sons of Ulster Marching Down the Somme $e^{15}$ ont largement contribué à ce changement, utilisant toutes les ressources de l'imagination et du verbe pour modifier notre vision et nous faire mieux revoir et comprendre les hommes et les faits du passé.

\section{NOTES}

1. Paul Ricœur, La mémoire, l'histoire, l'oubli, Paris, Seuil, coll. « Essais », 2000.

2. Voir par exemple Roy Foster, Modern Ireland : 1600-1972, London, Allen Lane, 1988.

3. " 1916 was an attempt to make failure glorious and memorable», Fintan O'Toole, "The Failure of Failure ", in Dermot Bolger (dir.), 16 on 16. 16 Irish Writers on the Easter Rising, Dublin, Raven Arts Press, 1988, p. 42.

4. Roddy Doyle, A Star Called Henry, London and New York, Penguin, 1999.

5. Paul Ricœur, op. cit., p. 104.

6. Sebastian Barry, A Long Long Way, London and New York, Viking, 2005. Tous les numéros de pages entre parenthèses renvoient à cette édition.

7. Sebastian Barry, The Steward of Christendom, London, Royal Court Theatre \& Methuen, 1995.

8. Sebastian Barry, Annie Dunne, London, Faber and Faber, 2002.

9. Sebastian Barry, Prayers of Sherkin, in Plays : 1, London, Methuen Drama, 1997.

10. Paul Ricœur, op. cit., p. 66.

11. Christina Hunt Mahony, «Barry's Naifs and the Poetry of Humanism », in Christina Hunt Mahony (dir.), Out of History. Essays on the Writings of Sebastian Barry, Washington, DC, The Catholic University of America Press, 2006.

12. Stephen Crane, The Red Badge of Courage (1895), New York, Pocket Books, 1976, p. 61.

13. René Girard, La violence et le sacré, Paris, Grasset, 1972.

14. Paul Ricœur, op. cit., p. 579.

15. Frank McGuiness, See the Sons of Ulster Marching Down the Somme, London, Faber, 1986. 


\section{RÉSUMÉS}

Dans A Long Long Way, publié en 2005, Sebastian Barry poursuit son œuvre de recouvrement de la mémoire nationale, entreprise dans ses pièces de théâtre, comme The Steward of Christendom, mais aussi dans ses romans comme Annie Dunne. En mettant successivement en scène les différents membres de la même famille, les Dunne, caractérisée par son appartenance à une classe sociale aujourd'hui rejetée dans les marges de l'histoire, celle des catholiques loyalistes, et ayant de nombreux points communs avec la propre famille de l'écrivain, Barry cherche à mettre en lumière les événements passés de l'Irlande volontairement laissées dans l'ombre par l'historiographie nationaliste. Le protagoniste de A Long, Long Way, Willie Dunne, permet ainsi à Barry de restituer l'expérience des milliers de soldats irlandais engagés sous l'uniforme britannique pendant la Première Guerre Mondiale, et auxquels le nouvel Etat irlandais refusa pendant longtemps de rendre hommage par des commémorations officielles, alors même que la rébellion de 1916 donna lieu à des cérémonies grandioses. En s'appuyant sur les notions d'oubli forcé ou de mémoire forcée, définies par Paul Ricœur dans La mémoire, l'histoire, l'oubli, cet article s'applique à analyser les procédés utilisés par Barry et à confronter la fiction aux faits historiques.

In A Long Long Way published in 2005, Sebastian Barry continues to explore the hidden recesses of Irish national history, as he did previously in his plays (The Steward of Christendom) or his novels ( Annie Dunne). Here he stages yet another member of the Dunne family, who, being Catholics loyal to the Crown, like Barry's own forefathers, are representative of a whole class generally excluded from nationalist historiography for reasons of non-conformity. Willie Dunne is one of the thousands of young Irishmen who enrolled in the British Army in WWI to fight in the Somme and in Flanders, and whose sacrifice was denied official recognition and tribute by the independent Irish State, until very recently, whereas the 1916 Rebellion was celebrated on a grand scale. Through the character of Willie, Barry questions the notion of sacrifice and its significance when it is not acknowledged by the community for whose sake it is performed. This article relies on Paul Ricœur's definitions of "forced oblivion" and «forced remembrance" to analyse the devices used by Barry and to compare fiction to historical facts.

\section{INDEX}

Mots-clés : Barry Sebastian, histoire et fiction, histoire et mémoire, bataille de la Somme (1916), Première Guerre mondiale, trauma

Keywords : history and fiction, battle of the Somme (1916), history and memory, trauma, Barry Sebastian, First World War 
AUTEUR

SYLVIE MIKOWSKI

Université de Reims-Champagne-Ardenne 